\title{
A new scoring system for simple risk prediction in patients with unprovoked venous thromboembolism
}

T he Vienna prediction model has been developed to assess risk of recurrence in patients who have experienced an unprovoked venous thromboembolism (VTE). This new riskscoring system is a simple nomogram that uses information about the patient's sex, location of VTE, and D-dimer levels to predict the likelihood that the patient will experience recurrent VTE.

Recurrent VTEs can be fatal. Risk of recurrence is low among patients whose VTEs were provoked by events such as trauma and surgery. Unprovoked VTE, however, is associated with a higher risk of recurrent events. Most recurrences can be prevented by antithrombotic therapy, but clinicians need to know which patients should be prescribed prophylactic treatment. Various methods for risk stratification are available; however, use of these approaches is often unfeasible in daily routine care of individual patients, owing to the cost and nature of the tests required to assess some of the included laboratory risk factors.

In an article based on the findings of almost 20 years of research, Sabine Eichinger and colleagues from the Medical University of Vienna, Austria, have developed and validated a new,

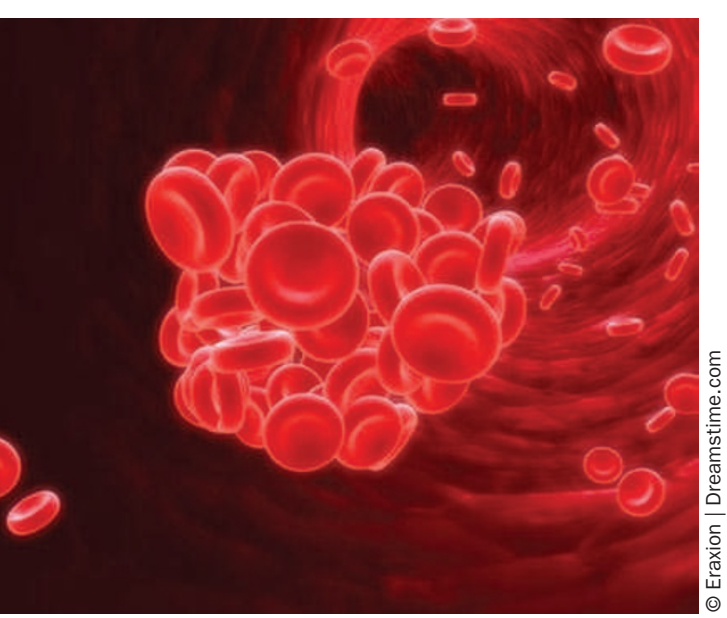

simple model for prediction of recurrent VTEs in patients presenting with their first, unprovoked VTE. The researchers initiated a prospective cohort studycalled the Austrian Study on Recurrent Venous Thromboembolism (AUREC) - in 1992 and, since then, almost 1,000 patients with a first, unprovoked VTE have been enrolled and followed after discontinuation of at least 3 months of oral anticoagulation therapy.

For the recent report in Circulation, the 929 patients were followed up for a median 43.3 months. During this time, 176 patients experienced symptomatic recurrent VTE (100 had deep-vein thromboses and 76 had pulmonary embolisms), and 3 of these patients died as a result of them. Recurrence was only classified as provoked (by surgery or trauma) in 16 of these patients. Kaplan-Meier analysis established that the cumulative probabilities of recurrence were $13.8 \%, 24.6 \%$, and $31.8 \%$ at 2,5 , and 10 years, respectively. In the first year of follow-up, the annual recurrence rate was $8.9 \%$; this rate reduced to $5.4 \%, 3.6 \%$, and $4.0 \%$ in the second, third, and fourth years, respectively.

The principle investigators, Sabine Eichinger and Paul Kyrle, preselected clinical and laboratory variables that had been independently confirmed to impact on the risk of VTE recurrence, and were reproducible and simple to assess. These variables included age, sex, BMI, location of VTE, in vitro thrombin generation, presence of the Factor V Leiden mutation, presence of the prothrombin G20210A mutation, and levels of D-dimer. A Cox proportional hazards model was used to analyze the preselected variables and only male sex, proximal deep-vein thrombosis, pulmonary embolism, and in vitro peak thrombin level were found to be statistically significant risk factors. However, when the investigators evaluated D-dimer without simultaneously considering in vitro peak thrombin level, D-dimer level was also found to be a statistically significant risk factor. Given that replacing in vitro peak thrombin level with D-dimer level did not affect the model's discrimination between patients at low and high risk of recurrent VTE, and because the D-dimer test is well standardized, Eichinger et al. chose to incorporate D-dimer level, male sex, proximal deep-vein thrombosis, and pulmonary embolism in their final model.

\section{4 ...clinicians need to} know which patients should be prescribed prophylactic treatment... 77

The risk model was validated using an internal procedure based on bootstrap validation. The c-statistics for the receiver operating characteristic curves for the scoring system's ability to predict VTE recurrence up to 12 and 60 months were 0.674 and 0.646 , respectively.

Dr Eichinger and colleagues now hope to validate their findings in independent patient cohorts. They are also planning a prospective study in which they will use the Vienna prediction model to determine the duration of anticoagulation therapy in patients with a first, unprovoked VTE. "At present," states Dr Eichinger, "prolonged anticoagulation (longer than 3 months) should be considered in all these patients. However, we believe that, by using our model, a substantial proportion of patients can be identified [as having] a recurrence risk ... low enough to consider shortterm anticoagulation [therapy] ... thereby avoiding an (unnecessary) bleeding risk."

\section{Bryony M. Mearns}

Original article Eichinger, S. et al. Risk assessment of recurrence in patients with unprovoked deep vein thrombosis or pulmonary embolism: the Vienna prediction model. Circulation 121, 1630-1636 (2010) 